

\title{
A new method for calculations of nanostructure kinetic stability at high temperature
}

\author{
A. S. Fedorov ${ }^{\mathrm{a}, \mathrm{b}, *}$, A. A. Kuzubov ${ }^{\mathrm{b}}$, M. A. Visotin ${ }^{\mathrm{b}}$, F. N. Tomilin ${ }^{\mathrm{a}, \mathrm{b}}$ \\ ${ }^{a}$ Kirensky Institute of Physics, Akademgorodok 50, Krasnoyarsk, 660036, Russia \\ ${ }^{b}$ Siberian Federal University, 79 Svobodny Prospect, Krasnoyarsk,660041, Russia
}

\begin{abstract}
A new universal method is developed for determination of nanostructure kinetic stability (KS) at high temperatures, when nanostructures can be destroyed by chemical bonds breaking due to atom thermal vibrations. The method is based on calculation of probability for any bond in the structure to stretch more than a limit value $L_{\max }$, when the bond breaks. Assuming the number of vibrations is very large and all of them are independent, using the central limit theorem, an expression for the probability of a given bond elongation up to $L_{m a x}$ is derived in order to determine the KS. It is shown that this expression leads to the effective Arrhenius formula, but unlike the standard transition state theory it allows one to find the contributions of different vibrations to a chemical bond cleavage. To determine the $\mathrm{KS}$, only calculation of frequencies and eigenvectors of vibrational modes in the groundstate of the nanostructure is needed, while the transition states are need not to be found. The suggested method was tested on calculating KS of bonds in some alkanes, octene isomers and narrow graphene nanoribbons of different types and widths at the temperature $\mathrm{T}=1200 \mathrm{~K}$. The probability of breaking of the $\mathrm{C}-\mathrm{C}$ bond in the center of these hydrocarbons is found to be significantly higher than at the ends of the molecules. It is also shown, that the KS of the octene isomers decreases when the double $\mathrm{C}=\mathrm{C}$ bond is moved to the end of the molecule, which agrees well with the experimental data. The KS of the narrowest graphene nanoribbons of different types varies by 1-2 orders of magnitude depending on the width and structure, while all of them are by several orders of magnitude less stable at high temperature than the hydrocarbons and benzene.
\end{abstract}

Keywords: stability nanostructures graphene nanoribbons

PACS: 05.70.Np 05.10.-a

\section{Introduction}

Nowadays, following the development of nanotechnologies, nanoelectronics, modern catalists, etc., a problem of determination of stability of different nanostructures as well as the surface of solids is becoming more and more topical. Estimates of thermodynamic stability, based on the Hibbs free energy $\mathrm{E}_{b i n d}$, are often fail to predict experimental yields of various nanostructures in their synthesis because of the importance of the factors that determine kinetic stability against cleavage of the nanostructure bonds. A classic example of this is permanent existence of diamond despite higher stability of the graphitic phase; another example is formation of buckminsterfullerene $\mathrm{C}_{60}$ in carbon-helium plasma.

\footnotetext{
${ }^{*}$ Corresponding author

Email address: alex99@iph.krasn.ru (A. S. Fedorov)
}

Although the $\mathrm{C}_{n}, n>60$, fullerenes have higher energies per atom, mainly $\mathrm{C}_{60}$ has the largest experimental yields.

Using different versions of molecular dynamics (MD) to determine $\mathrm{KS}$ is very computationally expensive, since it implies simulating systems for times, much longer than the inverse of characteristic thermal vibration frequencies $\left(\approx 10^{-13} \mathrm{~s}\right)$, which requires more than millions of standard timesteps ( $\approx 1$ femtosecond). For determination of KS of a system under transition from one stable state to another, one can calculate the frequency (or the probability) of the transition from on geometry to another with the help of standard transition state theory developed in (1), where the generalized Arrhenius equations is derived $v=v_{0} \exp \left(-\frac{E_{\text {barrier }}}{k T}\right)$, where $\mathrm{k}$ is the Boltzmann constant, $\mathrm{T}$ is the temperature, $E_{\text {barrier }}$ is the potential barrier for system passing from 
one stable state to another through a transitional state. The preexponential factor (the effective vibrational frequency) $v_{0}$ for an isolated structure is calculated with the well-known Eiring formula (1), which also considers zero-point energy:

$$
v_{0}=\frac{k T}{\hbar} \frac{\prod_{i=1}^{3 N-6}\left(1-\exp \left(-\frac{h v_{i}}{k T}\right)\right)}{\prod_{i=1}^{3 N-7}\left(1-\exp \left(-\frac{h v_{i}^{\prime}}{k T}\right)\right)}
$$

where $v_{i}^{\prime}$ are the vibrational frequencies of $\mathrm{N}$ atoms of the system in the saddle point, and $v_{i}$ - in the minimum.

Unfortunately, application of Arrhenius-type equation requires calculations of the transition barrier $E_{\text {barrier }}$ and determination of the systems potential energy surface. In the case of systems, consisting of hundreds of atoms, this becomes practically impossible, even if empirical potentials are used. The theory assumes that system overcomes the potential barrier along the path determined by only one (soft) vibrational mode, however it is quite obvious that cleavage of a bond may be caused by a combination of several vibrations.

At present there is no effective method for determination of kinetic stability of nanostructures as well as the surfaces of solids at high temperatures, when the chemical bonds can be broken due to atom thermal vibrations.

\section{Theory}

A new method to calculate KS based on the probabilities of any bond in the structure to stretch more than a limit value $L_{\text {max }}$, when the bond breaks, was suggested in our previous work (2). It is assumed that this probability describes the KS against destruction due to thermal vibrations. The proposed method was successfully used to calculate the KS of various fullerenes. In the present work we develop this approach for calculation of KS of carbonaceous heteroatomic molecules and nanostructures.

The approach is based on finding the probability for any chemical bond in the nanostructure to stretch more than a limit value of $L_{\max }$ with the help of central limit theorem (CLT) of probability theory. Assuming all of vibrational modes are independent and their number is large, CLT states that the probability of every atom displacement in every direction is distributed normally, thus the probability of a bond between any atoms elongation up to $L_{\max }$ can be calculated.

Then summing the contributions from harmonic vibration normal modes $k$ gives the displacement vector for every atom $n$ at time $t$ :

$$
\mathbf{R}(n, t)=\mathbf{R}_{0}(n)+\sum_{k=1}^{3 N-6} \mathbf{X}_{k}(n) \exp \left(i\left(\omega_{k} t+\phi_{k}\right)\right)
$$

where $\mathbf{R}_{0}(n)$ is the vector of $\mathrm{n}$-th atom equilibrium coordinates, $\omega_{k}$ is the $\mathrm{k}$-th vibration mode eigenfrequency, $\mathbf{X}_{k}(n)$ is the eigenvector of atom displacement in the kth vibration mode, and $\phi_{k}$ is the initial phase of the vibration mode. Hereinafter $3 N-6$ stands for the total number of the molecules vibration modes without translations and rotations. Apparently, the total energies of all atoms for a given mode are:

$$
E_{\text {pot }}=E_{\text {kin }}=\frac{m_{k}}{2} \sum_{n=1}^{N}\left|\mathbf{X}_{k}(n) \omega_{k}\right|^{2}=\frac{m_{k}}{2} \omega_{k}^{2} \mathbf{X}_{k}^{2},
$$

where $m_{k}$ is the reduced mass, and $\mathbf{X}_{k}$ is a vector of amplitudes in the space of atom numbers for the k-th mode. Using the Boltzmanns equipartition theorem, which gives the mean energy of one degree of freedom of a given mode, the expression for the vibration modes vector amplitude is easily derived:

$$
\left|\mathbf{X}_{k}\right|=\sqrt{\sum_{n=1}^{N}\left|\mathbf{X}_{k}(n)\right|^{2}}=\sqrt{\frac{k T}{m_{k} \omega_{k}^{2}}} .
$$

It is necessary to clarify the applicability of the three formulas stated above to describe the harmonic oscillation contribution at high temperatures $(T \approx 1000 \mathrm{~K})$ when the anharmonic contribution to the lattice vibrations can be considerable. Using (4), we can easily estimate the displacements of atoms within the harmonic approximation $|\Delta \mathbf{R}(n, m)|$. For $T=1000 K|\Delta \mathbf{R}(n, m)| \approx$ $0.4 \mathrm{~A}$. The contribution of anharmonic effects may be estimated from equation of thermal expansion $\frac{|\Delta \mathbf{R}(n, m)|}{|\mathbf{R}(n, m)|}=$ $\alpha T$, where $\alpha$ is the thermal expansion coefficient, which has typical values of $\alpha \sim 10^{-4} \div 10^{-5} K^{-1}$. Therefore, at $T=1000 K$ the mean changes of the chemical bond lengths due to anharmonicity is $\left|\Delta \mathbf{R}(n, m)_{\text {unharm }}\right| \sim$ $10^{-2} \div 10^{-1} \mathrm{~A}$, which is significantly smaller than the contribution from harmonic oscillations.

Further, having various vibration modes the timedependent vector connecting two atoms, $\mathrm{n}$ and $\mathrm{m}$, is equal to:

$$
\begin{aligned}
\mathbf{R}(n, m, t) & =\mathbf{R}_{0}(n m)+\Delta \mathbf{R}(n, m, t) \\
\Delta \mathbf{R}(n, m, t) & =\sum_{k=1}^{3 N-6}\left(\mathbf{X}_{k}(n)-\mathbf{X}_{k}(m)\right) \exp \left(i\left(\omega_{k} t+\phi_{k}\right)\right)
\end{aligned}
$$

As a first approximation of the displacements of the two atoms, after projecting the changes in the atom coordinates on the vector connecting the two atoms, the change in the distance between them is: 


$$
\begin{aligned}
\Delta \mathbf{R}(n, m, t)= & |\Delta \mathbf{R}(n, m, t)| \times \\
& \times \cos \left(\Delta \mathbf{R}\left(n, \widehat{m, t), \mathbf{R}_{0}}(n m)\right)\right.
\end{aligned}
$$

Given the displacements of every atom is a result of a big number of independent thermal vibration normal modes (5) and the contribution of each mode is relatively small, one can use CLT, which states that the distribution of atom displacements $\mathbf{X}(n)$, being a sum of small shifts $\mathbf{X}_{k}(n)$ by independent normal modes, represents a normal distribution D:

$$
\begin{array}{r}
D\left(\mathbf{X}(n)=\sum_{k=1}^{3 N-6} \mathbf{X}_{k}(n)\right)= \\
\frac{1}{\sqrt{2 \pi} \sigma} \exp \left(-\frac{(\mathbf{X}(n)-\langle\mathbf{X}(n)\rangle)^{2}}{2 \sigma^{2}}\right)
\end{array}
$$

where the mean atom displacement $\langle X(n)\rangle=0$, and the total dispersion of atom displacement $\sigma^{2}$ can be found from the Lindeberg CLT:

$$
\sigma^{2}=\sum_{k=1}^{3 N-6} \sigma_{k}^{2}
$$

The dispersion of $\mathrm{n}$-th atom displacements due to $\mathrm{k}$-th vibration normal mode is:

$$
\sigma_{k}^{2}(n)=\left\langle\left|\mathbf{X}_{k}(n) \exp \left(i\left(\omega_{k} t+\phi_{k}\right)\right)\right|^{2}\right\rangle=\left|\mathbf{X}_{k}(n)\right|^{2}
$$

Similar expression gives the dispersion of $\mathrm{n}$-th and $\mathrm{m}$-th atoms relative displacement:

$$
\begin{aligned}
\sigma_{k}^{2}(n, m) & =\left\langle\left|\left(\mathbf{X}_{k}(n)-\mathbf{X}_{k}(m)\right) \exp \left(i\left(\omega_{k} t+\phi_{k}\right)\right)\right|^{2}\right\rangle \\
& =\left|\mathbf{X}_{k}(n)-\mathbf{X}_{k}(m)\right|^{2}=\left|\mathbf{X}_{k}(n, m)\right|^{2}
\end{aligned}
$$

Using equations (5-10) and assuming that a chemical bond between two atoms $\mathrm{n}$ and $\mathrm{m}$ breaks if the distance between them becomes bigger than $L_{\max }$, an expression for probability $P_{n m}$ of the bond cleavage is:

$$
\begin{aligned}
P_{n m} & =P\left(|\mathbf{R}(n)-\mathbf{R}(m)|>X_{\max }\right) \\
& =\sum_{k=1}^{3 N-6} \frac{\left|\mathbf{X}_{k}(n, m)\right| \cos \left(\mathbf{X}_{k}\left(n, \widehat{m), \mathbf{R}_{0}}(n m)\right)\right.}{\sigma_{k}(n, m)}>X_{\max } \\
& =\frac{2}{\sqrt{\pi} \sigma(n, m)} \int_{X_{\max }}^{\infty} \exp \left(-\frac{u^{2}}{2 \sigma^{2}(n, m)}\right) d u \\
& =\frac{1}{2} \operatorname{erfc}\left(\frac{X_{\max }}{\sqrt{2} \sigma(n, m)}\right)
\end{aligned}
$$

where the total dispersion $\sigma^{2}(n, m)$ is calculated from (8), and $\operatorname{erfc}(x)$ is the complementary error function, which is decreasing rapidly and $\operatorname{erfc}(x) \approx 0$ for $x>2$. An expansion is known for such values:

$$
\operatorname{erfc}(x) \approx \frac{1}{\sqrt{2 \pi}} \exp \left(-\frac{x^{2}}{2}\right)\left(\frac{1}{x}-\frac{1}{x^{3}}\right)
$$

Using (11), the probability of bond cleavage is:

$P_{n m} \approx \frac{1}{\sqrt{2 \pi}} \exp \left(-\frac{X_{\max }^{2}}{2 \sigma^{2}(n, m)}\right) \frac{\sigma(n, m)}{X_{\max }}$

Using (4) and (9), the equation (12) is reduced to an expression that is similar to the Arrhenius equation for the probability of overcoming potential barrier $U_{\text {eff }}$ while breaking the bond:

$P_{n m} \approx A \sqrt{k T} \exp \left(-\frac{U_{\text {eff }}}{k T}\right)$

where the determinative exponential factor in (12) and the possibility to divide the total atom relative displacement dispersion into partial contributions from the independent vibration dispersions (8) make it possible to distinguish the contributions of each vibration mode to the probability of the given bond cleavage, which is important in analysis of the structures stability against different vibrati on modes.

\section{Results}

In this work, we have investigated KS, to be more precise, the probability of chemical bonds breaking at high temperature by example of normal (n-) and branched alkanes: n- $\mathrm{C}_{4} \mathrm{H}_{10}, \mathrm{C}_{6} \mathrm{H}_{14}$ (both normal and branched), n- $\mathrm{C}_{8} \mathrm{H}_{18}, \mathrm{n}-\mathrm{C}_{10} \mathrm{H}_{22}$, as well as zigzag and armchair narrow periodic graphene nanoribbons with the width of 3 , 4 , and 5 hexagons. The calculations of ground state geometric structure with vibration spectrum and the eigenvectors of the normal vibrations for the hydrocarbons molecules were conducted using the GAMESS code within the DFT formalism using B3LYB exchangecorrelation functional and cc $\mathrm{pVDZ}$ basis. This modern basis set has been specifically designed for post HartreeFock calculations, but it also works well for DFT calculations. For calculations of periodic graphene nanoribbons VASP 5.3.5 ((3), (4)) software package was used in combination with the PBE exchange-correlation functional and PAW formalism ((5), (6)). At the prestage, the geometry optimization of the nanoribbons was conducted, as well as the optimization of the length of the translational vector along the direction of their periodicity. To isolate the nanoribbons from their periodic images in the neighboring cells, which are necessarily 


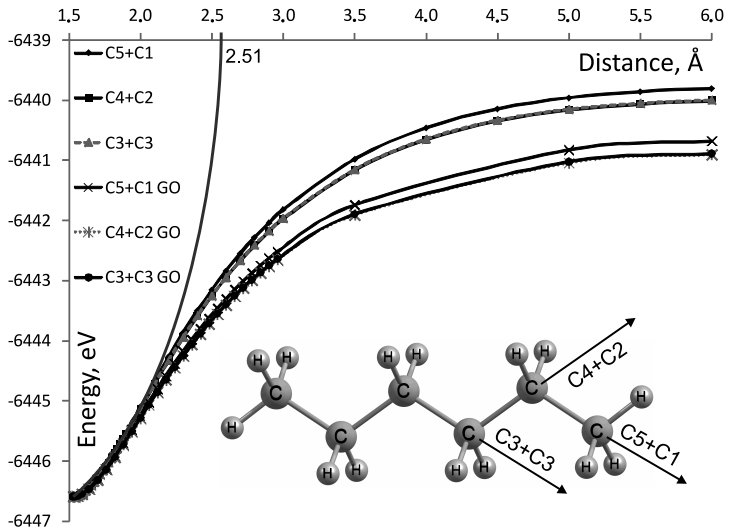

Figure 1: Dependence of the total energy in n- $\mathrm{C}_{6} \mathrm{H}_{14}$ on the extension of the corresponding bond. Lines marked as GO corresponds to the energies calculated with full optimization of both fragments.

present when the periodic boundary conditions are applied, vacuum spacings of $20 \AA$ were made along the normal to the nanoribbons plane and in the plane along a perpendicular to the nanoribbons directions. The stopping criteria for geometry optimization in all cases was that the maximal force acting on atoms were not more than $0.01 \mathrm{eV} / \AA$. Integrations on the first Brillouin zone (1BZ) were done on a $12 \times 1 \times 1$ Monkhorst-Pack (7) k-point grid. The vibration normal modes were calculated in the $\Gamma$ point only, within the analytical frozen phonon method by the density functional perturbation theory (DFPT) (8).

The maximum increase in length $L_{\max }$ of C-C bonds was defined as $0.98 \AA$. To find this value by the example of n-hexane $\mathrm{C}_{6} \mathrm{H}_{14}$, the total energy $U(r)$ profile was calculated while two $\mathrm{C}_{3} \mathrm{H}_{7}$ fragments were moved apart along the line connecting two carbon atoms inside these fragments, see Fig.1. During that, at each separation distance $r \leq 6 \AA$ both fragments were fully optimized. Having the energy profile, the potential barrier for chemical bond breaking $U_{p o t}=6.59 \mathrm{eV}$ was obtained.

Next, fitting the $U(r)$ profile (grey thin line in Fig.1) at small C-C bond extensions $\Delta r \leq 0.5 \AA$ with a quadratic function and using the value of $U_{\text {pot }}, L_{\text {max }}=$ $0.98 \AA$ A was finally obtained. In the same way, the value of $L_{\max }=1.05 \AA$ for the pair of $\mathrm{C}$ and $\mathrm{H}$ atoms was found from extension of the tertiary $\mathrm{C}-\mathrm{H}$ bond in $\mathrm{n}$ hexane. These values of $L_{\max }$ were used in all the calculations.

Apparently, while extending a chemical bond up to $L_{\max }$ before it breaks, the bond can be described as in an excited state. One of the features of the proposed method for KS determination is that there is no need to find the transition states of all bonds in the nanostructure, which is obviously very complex job. Instead of it, we use $L_{\max }$ obtained from reference systems. The proposed method was used to calculate the data on the $\mathrm{KS}$ of alkane molecules, the results are summarized in Table 1. The temperature in the calculations was set to $1200 \mathrm{~K}$, the temperature of the onset of hydrocarbon thermal dissociation.

The table displays probabilities of C-C and $\mathrm{C}-\mathrm{H}$ bonds breaking, as well as the corresponding interatomic distances, in the center and at the edges of molecules. It can be seen that for all the molecules the probability of destroying the least stable bonds $(\mathrm{C}$ C) in the center of the molecules is significantly higher than probability of breaking the bonds at the edges. The difference is bigger for the longer carbon chains; this agrees well with available experimental data on the stability of n-alkanes with different chain lengths (9). The same relation can be seen for the $\mathrm{C}-\mathrm{H}$ bonds. In the branched isohexane, the probability of the central $\mathrm{CH}$ group tearing off the central carbon atom is two orders of magnitude higher than probability of breaking of the central C-C bond in n-hexane. The same behavior is observed for the $\mathrm{C}-\mathrm{H}$ bond with only one order of magnitude difference. In all the cases, the probability of bond cleavage strongly correlates with the bond length the longer the interatomic distance, the higher the probability of bond breaking.

While analyzing the KS of the alkanes, it was found that the decisive role in the molecule destruction falls not on a single, but on a group of some valent vibrations that have maximum contribution in the extension/compression of the weakest bond. For example, the main role of $\mathrm{C}-\mathrm{C}$ bonds cleavage in the hexane $\mathrm{C}_{6} \mathrm{H}_{14}$ is shared between six different vibrations.

The same approach was applied to KS evaluation for narrow periodic graphene nanoribbons with their edges terminated by hydrogen atoms. It was shown for $\mathrm{T}$ $=1200 \mathrm{~K}$ that probabilities of breaking of the $\mathrm{C}-\mathrm{H}$ bonds on the edges of armchair nanoribons with the widths of 3, 4, 5 hexagons (ANR7, ANR8 and ANR9 in standard notation) are equal to $3.48 * 10^{-24}, 4.86 *$ $10^{-23}, 2.32 * 10^{-22}$, respectively. The corresponding values for zigzag type nanoribbons (ZNR4, ZNR5 and ZNR6 in standard notation) are $2.81 * 10^{-24}, 4.65 *$ $10^{-22}, 5.96 * 10^{-20}$, respectively. At the same time, the probabilities of breaking of the $\mathrm{C}$ - $\mathrm{C}$ bonds in all considered nanoribbons were significantly lower than $\approx 10^{-32}$. The obtained results claim that the KS of the narrowest armchair nanoribbons of 3 hexagon width are significantly (1-2 orders of magnitude) higher than the KS of the nanoribbons with bigger, 4 or 5 hexagons, width. 
Table 1: Bond length $(\mathrm{d})$ and probability of breaking $(v)$ for bonds in the center and at the edge of different hydrocarbons

\begin{tabular}{|c|c|c|c|c|c|c|c|c|}
\hline $\mathrm{T}=1200 \mathrm{~K}$ & \multicolumn{2}{|c|}{ C- (center) } & \multicolumn{2}{c|}{ C- (edge) } & \multicolumn{2}{c|}{ C-H (center) } & \multicolumn{2}{c|}{ C-H (edge) } \\
\hline & $v$ & $\mathrm{~d}$ & $v$ & $\mathrm{~d}$ & $v$ & $\mathrm{~d}$ & $v$ & $\mathrm{~d}$ \\
\hline$C_{4} H_{10}$ & $6.04 * 10^{-29}$ & 1.533 & $1.65^{*} 10^{-29}$ & 1.531 & $3.01^{*} 10^{-35}$ & 1.106 & $4.34^{*} 10^{-36}$ & 1.103 \\
\hline$C_{6} H_{14}$ & $6.10^{*} 10^{-29}$ & 1.533 & $2.02^{*} 10^{-29}$ & 1.531 & $4.94^{*} 10^{-35}$ & 1.107 & $2.00^{*} 10^{-36}$ & 1.102 \\
\hline$C_{6} H_{14}$ izo & $1.66^{*} 10^{-27}$ & 1.542 & $4.39^{*} 10^{-29}$ & 1.532 & $3.15^{*} 10^{-34}$ & 1.109 & $5.63 * 10^{-36}$ & 1.104 \\
\hline$C_{8} H_{18}$ & $6.47^{*} 10^{-29}$ & 1.533 & $1.87^{*} 10^{-29}$ & 1.531 & $4.57^{*} 10^{-35}$ & 1.107 & $4.44 * 10^{-36}$ & 1.104 \\
\hline$C_{10} H_{18}$ & $1.67 * 10^{-27}$ & 1.543 & $5.17^{*} 10^{-29}$ & 1.532 & $6.53 * 10^{-35}$ & 1.107 & $3.01 * 10^{-36}$ & 1.103 \\
\hline
\end{tabular}

In addition to this, the KS of zigzag nanoribbons is an order of magnitude lower than the KS of armchair nanoribbons.

In order to compare the stability of the nanoribbons with stable hydrocarbons, having hydrogen atoms at the molecule edge, the KS of benzene molecule was calculated as well. At $\mathrm{T}=1200 \mathrm{~K}$ the probabilities of breaking of the $\mathrm{C}$ - $\mathrm{H}$ bonds equals to $0.27 * 10^{-36}$, which indicates that nanoribbon thermal stability at high temperature is inferior relative to benzene.

Summing up, a universal method is developed for determination of nanostructure kinetic stability at high temperatures, when the nanostructure gets destroyed by chemical bonds breaking due to atom thermal vibrations. It is shown that the method gives an effective Arrhenius formula. The examples of several normal and branched alkanes show good correlation of calculated KS with available experimental data. The method needs only the spectrum of normal vibrations to be known, and allows one to distinguish the contributions of each vibration mode to the KS of the given chemical bond.

\section{Acknowledgments}

The work was supported by the Russian Foundation for Basic Research grant N15-02-06869A. The authors would like to thank Joint Supercomputer Center of RAS, Moscow for the access to the MVS 100K cluster and Institute of Computational Modelling of SB RAS, Krasnoyarsk for providing the computational resources.

\section{References}

[1] H Eyring, D. Henderson, B.J. Stover and E.M. Eyring, Statistical Mechanics and Dynamics, John Wiley, New York, 1964, p.369

[2] A. S. Fedorov, D. A. Fedorov, A. A. Kuzubov, P. V. Avramov, Y. Nishimura S. Irle and Henryk A. Witek, Phys.Rev. Lett., 107 No.17 (2011) 75506-1.

[3] G. Kresse and J. Hafner, Phys. Rev. B47 (1993) RC558 .

[4] G. Kresse and J. Furthmüller, Phys. Rev. B54 (1996) 11169.

[5] Blochl, P. E. Phys. Rev. B50 (1994) 17953-17979.

[6] Kresse, G. Phys. Rev. B59 (1999) 17581775
[7] Monkhorst, H.J. Phys. Rev. B13 (1976) 51885192.

[8] Gonze, X. Lee, C. Phys. Rev. B 55 (1997) 10355-10368.

[9] http://www.neftemagnat.ru/enc/296. 